\title{
Sentinel Lymph Node Biopsy Prior to Neoadjuvant Chemotherapy: A Series of 89 Patients
}

\author{
Flipo Bernard $^{1 \#}$, Clough Krishna², Nos Claude ${ }^{3}$, Miramand Bernard ${ }^{4}$, Michel Cecile ${ }^{5}$, \\ Houvenaeghel Gilles ${ }^{6}$, Meurette Jacques $^{7}$, Alfonsi Jean-Pierre ${ }^{8}$ \\ ${ }^{1}$ Breast Surgery Department, Centre Antoine Lacassagne, Nice, France; ${ }^{2}$ Clough Krishna, Institut du Sein, Paris, France; ${ }^{3}$ Nos Claude, \\ Institut du Sein, Paris, France; ${ }^{4}$ Miramand Bernard, Polyclinique Urbain V, Avignon, France; ${ }^{5}$ Michel Cecile, Biostatistics \\ Department, Centre Antoine Lacassagne, Nice, France; ${ }^{6}$ Houvenaeghel Gilles, Institut Paoli-Calmettes, Marseille, France; ${ }^{7}$ Meurette \\ Jacques, Clinique Ste-Amé, Lambres lez Douai, France; ${ }^{8}$ Alfonsi Jean-Pierre, Clinique St. Antoine, Ville di Pietrabugno, France. \\ Email: " bernard.flipo@nice.unicancer.fr
}

Received August $2^{\text {nd }}, 2012$; revised September $4^{\text {th }}, 2012$; accepted September $15^{\text {th }}, 2012$

\begin{abstract}
Purpose: There are still un-responded questions concerning the type of and the timing of axillary procedures that has to be performed in association with neoadjuvant chemotherapy. Methods: We led a prospective, multicentric, non-randomized study to evaluate the feasibility and accuracy of sentinel lymph node biopsy before neoadjuvant chemotherapy. The clinical and radiological response to chemotherapy was evaluated after 4 treatment cycles and at the end of chemotherapy. Axillary lymph node dissection was performed 3 to 4 weeks after chemotherapy. Histological analysis of sentinel lymph node biopsies and axillary lymph node dissections were studied for each patient. Results: Eighty nine patients had sentinel lymph node biopsy. The identification rate for sentinel lymph nodes was $98.9 \%$. The sentinel lymph node biopsies were metastatic in 44 of 88 patients. Axillary lymph nodes were metastatic in 12 cases. The negative predictive value was $91.1 \%$ [95\% CI: $85.1 \%$ - 97.1\%]. Conclusion: Identification rate and negative predictive value of sentinel lymph node biopsy prior to neoadjuvant chemotherapy confirm that the procedure is suitable with its use in standard practice. This approach comprises two surgical procedures, but allows a better nodal status evaluation.
\end{abstract}

Keywords: Breast Cancer; Neoadjuvant Therapy; Clinical Trial; Sentinel Lymph Node Biopsy

\section{Introduction}

The recourse to neoadjuvant chemotherapy (NC) has increased during the last years as it sometimes allows breast conservation and permits an evaluation of tumor response though providing prognostic information. However, even if the side effects of axillary dissection [1], as well as the benefits of Sentinel Lymph Node Biopsy (SLNB) are known [2], there are still un-responded questions concerning the type of and the timing of axillary procedures that has to be performed in association with NC [3]. A meta-analysis has shown that identification rates for SLNB after neoadjuvant chemotherapy, are suitable with its use in standard practice [4]. Moreover, Jones et al. and the meta-analysis of Iwase et al. found a significantly better identification rates when SLNB was performed before neoadjuvant chemotherapy (96\% $100 \%$ versus $71 \%-98 \%$ ), and though recommended the procedure before $\mathrm{NC}$ for clinically N0 patients $[5,6]$. Last, it may give data useful in guiding systemic [7], radiation

\footnotetext{
*Conflict of Interest: Authors have no conflict of interest.
}

${ }^{\#}$ Corresponding author. and surgical treatment decisions. These elements led us to design a study consisting to a SLNB before NC and an axillary lymph node dissection (AD) after NC, in order to validate the feasibility, accuracy and to evaluate the interest of SLNB prior to neoadjuvant chemotherapy.

\section{Patients and Methods}

\subsection{Study Design}

We led a prospective, multicentric, non-randomized study. This study obtained authorization from French Health authorities. It was designed and conducted in accordance with Good Clinical Practice Guidelines and the latest revision of the declaration of Helsinki. The ethics committee (CPP Sud Mediterranee V) approved the study in April 2006. Before inclusion, all patients provided written informed consent.

\subsection{Patients}

The inclusion criteria retained were: age $\geq 18$ and $\leq 70$ years-old, histological proof of single, unilateral, T2 or 
T3 invasive breast cancer according to AJCC classification [8] and/or tumor size not permitting breast conservative surgery, clinical and radiological N0. Exclusion criteria included: distant metastasis, inflammatory breast cancer, previous chemotherapy for contralateral breast cancer, and allergy to Patent Blue dye.

\subsection{Lymphatic Mapping of the Sentinel Node}

All patients had preoperative periareolar or peritumoral radiocolloid ( ${ }^{99}$ Technetium) and Patent Blue dye injection. A lymphoscintigraphy of the breast and the axilla were performed to determine the position of the sentinel node as described by Lelievre et al. [9].

\subsection{Sentinel Lymph Node Biopsy}

SLNB was performed at the time of chemotherapy-port insertion under general anesthesia. All lymph nodes presenting blue dye and/or radioactive counts were identified as sentinel lymph nodes, removed and sent individually for histological analysis with information concerning blue dye uptake and ex-vivo radioactivity count.

\subsection{Neoadjuvant Chemotherapy}

All patients were assigned to receive 5-fluorouracil 500 $\mathrm{mg} / \mathrm{m}^{2}$, epirubicin $100 \mathrm{mg} / \mathrm{m}^{2}$, and cyclophosphamide $500 \mathrm{mg} / \mathrm{m}^{2}$ (FEC) intravenously on day 1 every 21 days for 4 cycles followed by docetaxel $100 \mathrm{mg} / \mathrm{m}^{2}$ intravenously on day 1 every 21 days for an additional 4 cycles. Human epidermal growth factor receptor 2 (Her2) positive patients also received trastuzumab concomitant to docetaxel. The clinical and radiological responses to $\mathrm{NC}$ were evaluated after 4 treatment cycles and at the end of chemotherapy treatment.

In this study, clinical response was defined as: complete response, no palpable tumor; partial response, regression of $\geq 50 \%$ of tumor; stabilization, regression of $<50 \%$ of tumor or no change at all; progression, increase of the tumor size.

\subsection{Axillary Lymph Node Dissection and Breast Surgery}

Breast surgery was performed 3 to 4 weeks after the end of neoadjuvant chemotherapy. The surgical procedures comprised radical mastectomy or breast-conserving treatment according to tumor response. Level I and II axillary lymph node dissections were systematically performed.

\subsection{Histology}

Sentinel nodes were fixed with formalin, embedded in paraffin, and analyzed by serial sectioning $(200 \mu \mathrm{m})$, stained with hematoxylin and eosin, and by immunohis- tochemistry, if suspicious cells were found or if nodes were negative. Axillary lymph node dissection and tumor were examined according to standard procedures. The histological response of the tumor and nodes was evaluated by using Sataloff classification [10].

\subsection{Statistical Analysis}

The identification rate (IR) was defined as the proportion of procedures in which a sentinel lymph node was successfully identified.

The negative predictive value (NPV) was defined as true negative $(\mathrm{TN}) /(\mathrm{TN}+$ False Negative). In this setting, a false-negative SLN could have resulted either from misidentification of the SLN or from disease progression under chemotherapy in 1 or more non-SLNs.

\section{Results}

\subsection{Patients}

From February 2006 to September 2009, 89 patients were included at 11 centers. The mean age was 50.9 years (range 28 - 77, SD = 10.6). Mean radiologic tumor size was 3.1 centimeters (range $0.8-6.6, \mathrm{SD}=1.1$ ). Patient characteristics are described in Table 1.

\subsection{Sentinel Lymph Node Biopsy}

The identification rate for sentinel lymph node was $98.9 \%$ (88 out of 89 procedures). Blue dye and radio colloids were used in combination in 77 patients $(86.5 \%)$. Five patients $(5.6 \%)$ received only blue dye and 7 only radio colloid $(7.9 \%)$. The only patient for whom sentinel lymph node identification failed had both blue dye and radio colloids injections. The mean number of nodes removed per procedure was 2.8 (range 1 - 9; SD 1.6). The SLNB were metastatic in 44 of 88 patients $(50 \%)$. Sentinel nodes were found in external mammary chain in 82 patients, in external and internal mammary chain in 3 patients, in internal mammary chain only, in 1 patient and in other location in 1 patient.

\subsection{Neoadjuvant Chemotherapy}

NC was completed in 83 patients (93.3\%): 48 (57.8\%) patients completed 8 cycles and $35(42.2 \%)$ completed 6 cycles. The 6 remaining patients $(6.7 \%)$ completed at least 3 cycles chemotherapy due to progression $(n=3)$, major side effects $(n=2)$ or patient decision $(n=1)$.

Out of the 83 patients that completed neoadjuvant chemotherapy, clinical response was complete in 21 patients $(25.3 \%)$ and partial in 55 patients (66.3\%) (Table 2).

\subsection{Surgical Procedure}

Breast conservative procedure was performed in 65 pa- 
Table 1. Patient characteristics.

\begin{tabular}{|c|c|c|}
\hline Characteristics $(89,100 \%)$ & Number of patients (n) & $\%$ \\
\hline \multicolumn{3}{|l|}{ Age (years) } \\
\hline Median [range] & 50 & {$[28-77]$} \\
\hline$\leq 50$ & 47 & $52.8 \%$ \\
\hline$>50$ & 42 & $47.2 \%$ \\
\hline \multicolumn{3}{|l|}{ Tumor size $^{*}$ (cm) } \\
\hline Median [range] & 3.0 & {$[0.8-6.6]$} \\
\hline \multicolumn{3}{|l|}{ Clinical stage } \\
\hline $\mathrm{cT} 2$ & 75 & $84.3 \%$ \\
\hline $\mathrm{cT} 3$ & 14 & $15.7 \%$ \\
\hline \multicolumn{3}{|l|}{ Histology } \\
\hline Invasive ductal cancer & 85 & $95.5 \%$ \\
\hline Invasive lobular cancer & 1 & $1.1 \%$ \\
\hline Others & 3 & $3.4 \%$ \\
\hline \multicolumn{3}{|l|}{ Tumor grade (SBR) } \\
\hline I & 9 & $10.1 \%$ \\
\hline II & 34 & $38.2 \%$ \\
\hline III & 40 & $44.9 \%$ \\
\hline Unknown & 6 & $6.7 \%$ \\
\hline \multicolumn{3}{|l|}{ Hormonal receptor status } \\
\hline Estrogen-Receptor+ & 57 & $64.0 \%$ \\
\hline Estrogen-Receptor- & 30 & $33.7 \%$ \\
\hline Estrogen-Receptor unknown & 2 & $2.3 \%$ \\
\hline Progesterone-Receptor + & 45 & $50.6 \%$ \\
\hline Progesterone-Receptor- & 41 & $46.1 \%$ \\
\hline Progesterone-Receptor unknown & 3 & $3.3 \%$ \\
\hline HER2 overexpression & 26 & $29.2 \%$ \\
\hline
\end{tabular}

*Radiologic.

tients (73\%), while 24 (27\%) patients had a radical mastectomy. All patients underwent AD. The mean number of nodes obtained per axillary dissection was 9.4 (range 1 37 ; $\mathrm{SD}=4.4$ ). Nodes were metastatic (either micro metastatic or macro metastatic) in 12 cases (13.5\%). Among the 44 patients who had a metastatic SLNB, 8 had confirmed metastatic nodes on AD (Table 3). The negative predictive value was $91.1 \%$ [95\% CI: $85.1 \%$ - 97.1\%]. The nodes of the patient whose SLNB was not identified were not metastatic.

Four patients had a negative SLNB and a positive axillary dissection (9.1\% of total negative SLNB). Two
Table 2. Clinical and histological responses.

\begin{tabular}{lcc}
\hline & $\begin{array}{c}\text { NC complete } \\
(\mathbf{n}=\mathbf{8 3})\end{array}$ & $\begin{array}{c}\text { NC incomplete } \\
(\mathbf{n}=\mathbf{6})\end{array}$ \\
\hline Clinical response & 21 & - \\
Complete & 55 & 2 \\
Partial & 7 & 1 \\
Stabilization & - & 3 \\
Progression & & \\
Histological response & & \\
Sataloff & 34 & - \\
T-A & 30 & 1 \\
T-B & 17 & 2 \\
T-C & 2 & - \\
T-D & & \\
Sataloff & & 5 \\
N-A & 46 & - \\
N-B & 25 & \\
N-C & 8 & \\
N-D & & \\
\hline
\end{tabular}

NC: neoadjuvant chemotherapy.

Table 3. Concordance between histological analysis of SLNB before NC and axillary lymph node dissection after NC.

\begin{tabular}{lccc}
\hline AD results & Metastatic & Non metastatic & Total \\
\hline MLNB results & $8(18.2 \%)$ & $36(81.8 \%)$ & 44 \\
Non metastatic & $4(9.1 \%)$ & $40(90.9 \%) 1^{*}$ & $441^{*}$ \\
Total & 12 & 77 & 89 \\
\hline $\begin{array}{l}\text { 1 non identified SLN; SLNB: sentinel lymph node biopsy; AD: axillary } \\
\text { dissection. }\end{array}$
\end{tabular}

patients have over 50 years and two are under 50 years. The mean radiological tumor size was 1.7 centimeters (range 1.5 - 2.0). All patients presented an invasive ductal cancer and a cT2 clinical stage. Three out of four patients had a tumor grade II (SBR). Estrogen and progesterone receptor status were positive for 3 patients. Her2 status was negative for 3 out of 4 patients. Of note, one patient presented a triple-negative breast cancer. The mean number of SLN was $2.75 \%$ and $100 \%$ were localized in external mammary chain. All four patients received at least 6 chemotherapy treatment cycles. The residual tumor size ranged from 0 to 2.5 centimeters.

Eighty two percent of the patients who had a positive SLNB did not have any other metastatic node in the axil- 
lary dissection.

\section{Discussion}

Sentinel lymph node biopsy in breast cancer is a highly sensitive technique for identifying axillary metastasis in a minimally invasive manner, compared to standard axillary dissection [11]. The use of this technique in association with $\mathrm{NC}$ is controversial and the optimal timing has not yet been precisely determined.

However, there are several arguments that are favorable to the use of SLNB prior to NC. A recent review article has shown that identification rates for SLNB prior to $\mathrm{NC}$ are suitable with its use in standard practice [12]. This is confirmed by our findings: the identification rate in our study reached $98.9 \%$. Moreover, several studies have demonstrated an identification rates significantly better when SLNB was performed before neoadjuvant chemotherapy $(96 \%$ to $100 \%)$ rather than after $(71 \%$ to $98 \%$ ), and though recommended the procedure before $\mathrm{NC}$ for clinically N0 patients $[5,6]$. The lower identification rate after $\mathrm{NC}$ could be related to chemotherapy-related fibrosis and tumor debris that change the lymphatic flow of the breast $[12,13]$. Moreover, if NC eradicates metastatic foci in SLNs but not in non-SLNs, falsenegative results will eventually occur. However, in this setting in which axillary nodal status was affected by NC, a precise false-negative rate could not be calculated.

In our study, we should notice that among the 4 patients with negative SLN and positive AD, 2 showed progression of the disease under NC. Though, these 2 patients were not adequate for the SLNB evaluation prior to NC as the positive could be related to the progression of the disease. Moreover, the false negative rates of SNLB after $\mathrm{NC}$ found in the literature seem to be higher than those of SNLB prior to NC. In addition, considering the fact that nodal down staging occurs in $20 \%$ to $40 \%$ of cases after $\mathrm{NC}$ [14], the nodal status, which is a major prognosis parameter, might be distorted if explored after NC.

Last, knowing the nodal status is helpful when planning breast surgery as we might determine whether radiotherapy is required. This can affect the decision of achieving or postponing breast reconstruction when a mastectomy is planned.

Pre-NC evaluation of the nodal status goes along with the recent published data concerning SLNB. Indeed, Krag et al. have shown that patients with negative SLN that undergo chemotherapy do not benefit from complementary $\mathrm{AD}$ in terms of axillary recurrence or survival [15]. We might though be able to spare axillary lymph nodes in patients that have a negative pre-NC SLNB [3]. When the pre-NC SLNB is positive, it is still recommended to perform AD. However, there are few publications that propose a second SLNB [16], which could be done after NC. The "second SLNB" evaluation is in process and it cannot yet be recommended in common practice. Otherwise, Giuliano et al. have shown that patients with positive SLNB that undergo chemotherapy do not benefit from AD [17]. These findings, if confirmed by other studies, might lead to lymph node sparing even in patients with positive SLNB that undergo chemotherapy. As the evolution of axillary procedures seems to head toward SLNB without AD for a majority of patients, we do believe that having a non-distorted pre-NC nodal status is better for prognosis assessment.

However, several remarks have to be made. The rate of positive SLNB in our series is high $(50 \%)$. This could be related to the fact that patients did not have ultrasound exploration of the axilla, which can help reducing the rate of positive SNLB [18]. We have though started systematic ultrasound exploration of the axilla prior to SLNB.

Moreover, our approach comprises two operations, before and after NC, while patients that undergo SLNB after NC sustain only one surgical procedure. Nevertheless, the SLNB was achieved at the time of chemotherapy-port insertion and the operating time was short as we did not have to wait for lymph node intra-operative histological examination.

\section{Conclusion}

This study evaluating nodal status for 89 patients before neoadjuvant chemotherapy constitutes the largest series of SLNB pre-NC. Our results seem to validate SNLB prior to $\mathrm{NC}$ by identification rate and negative predictive value suitable with its use in standard practice. Even if this approach comprises two surgical procedures, we do believe that it allows a better nodal status evaluation, as it is not distorted by chemotherapy. Basing our judgment on the results of the NSABP-B32 study [15], we could spare AD if the pre-NC SNLB is negative in patients who complete $\mathrm{NC}$ without disease progression.

\section{Acknowledgements}

This work was carried out with the participation of Dr. Monique Cohen, Dr. Jean-Claude Darmon, Dr. Sylvia Giard, Dr. Raoul Payan, Dr. Gérard Priou, Dr. Eric Walleghem, Dr. Ana Lorena Vasquez-Guerrero and Pr. Moïse Namer for the inclusion of patients.

This work was supported by Sanofi-Aventis and Cercle Sainte Agathe. ALVG's participation is possible thanks to a grant received by CONACYT (National Council of Science and Technology, Mexico).

\section{REFERENCES}

[1] J. M. Ververs, R. M. Roumen, A. J. Vingerhoets, et al., 
"Risk, Severity and Predictors of Physical and Psychological Morbidity after Axillary Lymph Node Dissection for Breast Cancer," European Journal of Cancer, Vol. 37, No. 8, 2001, pp. 991-999. doi:10.1016/S0959-8049(01)00067-3

[2] U. Veronesi, G. Paganelli, G. Viale, et al., "A Randomized Comparison of Sentinel-Node Biopsy with Routine Axillary Dissection in Breast Cancer," New England Journal of Medicine, Vol. 349, No. 6, 2003, pp. 546-553. doi:10.1056/NEJMoa012782

[3] J. P. Menard, J. M. Extra, J. Jacquemier, et al., "Sentinel Lymphadenectomy for the Staging of Clinical Axillary Node-Negative Breast Cancer before Neoadjuvant Chemotherapy," European Journal of Surgical Oncology, Vol. 35, No. 9, 2009, pp. 916-920. doi:10.1016/j.ejso.2008.11.002

[4] Y. Xing, M. Foy, C. D. Cox, et al., "Meta-Analysis of Sentinel Lymph Node Biopsy after Preoperative Chemotherapy in Patients with Breast Cancer," British Journal of Surgery, Vol. 93, No. 5, 2006, pp. 539-546. doi:10.1002/bjs.5209

[5] H. Iwase, Y. Yamamoto, T. Kawasoe, et al., "Advantage of Sentinel Lymph Node Biopsy before Neoadjuvant Chemotherapy in Breast Cancer Treatment," Surgery Today, Vol. 39, No. 5, 2009, pp. 374-380. doi:10.1007/s00595-008-3880-x

[6] J. L. Jones, K. Zabicki, R. L. Christian, et al., "A Comparison of Sentinel Node Biopsy before and after Neoadjuvant Chemotherapy: Timing Is Important," The American Journal of Surgery, Vol. 190, 2005, pp. 517-520. doi:10.1016/j.amjsurg.2005.06.004

[7] G. Bonadonna, P. Valagussa, A. Moliterni, et al., "Adjuvant Cyclophosphamide, Methotrexate, and Fluorouracil in Node-Positive Breast Cancer: The Results of 20 Years of Follow-Up," New England Journal of Medicine, Vol. 332, No. 14, 1995, pp. 901-906. doi:10.1056/NEJM199504063321401

[8] S. E. Singletary, C. Allred, P. Ashley, et al., "Staging System for Breast Cancer: Revisions for the 6th Edition of the AJCC Cancer Staging Manual," Surgical Clinics of North America, Vol. 83, No. 4, 2003, pp. 803-819.

[9] L. Lelievre, G. Houvenaeghel, M. Buttarelli, et al., "Value of the Sentinel Lymph Node Procedure in Patients with Large Size Breast Cancer," Annals of Surgical Oncology, Vol. 14, No. 2, 2007, pp. 621-626. doi:10.1245/s10434-006-9232-4

[10] D. M. Sataloff, B. A. Mason, A. J. Prestipino, et al.,
"Pathologic Response to Induction Chemotherapy in Locally Advanced Carcinoma of the Breast: A Determinant of Outcome," Journal of the American College of Surgeons, Vol. 180, No. 3, 1995, pp. 297-306.

[11] F. J. Fleming, D. Kavanagh, T. B. Crotty, et al., "Factors Affecting Metastases to Non-Sentinel Lymph Nodes in Breast Cancer," Journal of Clinical Pathology, Vol. 57, No. 1, 2004, pp. 73-76. doi:10.1136/jcp.57.1.73

[12] H. M. Kuerer, A. A. Sahin, K. K. Hunt, et al., "Incidence and Impact of Documented Eradication of Breast Cancer Axillary Lymph Node Metastases Before Surgery in Patients Treated with Neoadjuvant Chemotherapy," Annals of Surgery, Vol. 230, No. 1, 1999, pp. 72-78. doi:10.1097/00000658-199907000-00011

[13] K. Shimazu and S. Noguchi, "Sentinel Lymph Node Biopsy before versus after Neoadjuvant Chemotherapy for Breast Cancer," Surgery Today, Vol. 41, No. 3, 2011, pp. 311-316. doi:10.1007/s00595-010-4404-Z

[14] B. T. Hennessy, G. N. Hortobagyi, R. Rouzier, et al., "Outcome after Pathologic Complete Eradication of Cytologically Proven Breast Cancer Axillary Node Metastases Following Primary Chemotherapy," Journal of Clinical Oncology, Vol. 23, No. 36, 2005, pp. 9304-9311. doi:10.1200/JCO.2005.02.5023

[15] D. N. Krag, S. J. Anderson, T. B. Julian, et al., "Sentinel-Lymph-Node Resection Compared with Conventional Axillary-Lymph-Node Dissection in Clinically NodeNegative Patients with Breast Cancer: Overall Survival Findings from the NSABP B-32 Randomised Phase 3 Trial," The Lancet Oncology, Vol. 11, 2010, pp. 927-933. doi:10.1016/S1470-2045(10)70207-2

[16] E. R. Port, J. Fey, M. L. Gemignani, et al., "Reoperative Sentinel Lymph Node Biopsy: A New Option for Patients with Primary or Locally Recurrent Breast Carcinoma," Journal of the American College of Surgeons, Vol. 195, No. 2, 2002, pp. 167-172. doi:10.1016/S1072-7515(02)01268-1

[17] A. E. Giuliano, K. K. Hunt, K. V. Ballman, et al., "Axillary Dissection vs No Axillary Dissection in Women with Invasive Breast Cancer and Sentinel Node Metastasis: A Randomized Clinical Trial," JAMA, Vol. 305, No. 6, 2011, pp. 569-575. doi:10.1001/jama.2011.90

[18] B. P. Baruah, A. Goyal, P. Young, et al., "Axillary Node Staging by Ultrasonography and Fine-Needle Aspiration Cytology in Patients with Breast Cancer," British Journal of Surgery, Vol. 97, No. 5, 2010, pp. 680-683. doi: $10.1002 /$ bjs. 6964 